\title{
Virtual microscopy
}

\section{Lundin, J Lundin, J Isola}

\section{Applications in diagnostic pathology}

P n this issue of the Journal of Clinical Pathology, we describe an atlas of breast histopathology, implemented with web based virtual microscopy. ${ }^{1}$ The article highlights the advantages of virtual microscopy compared with traditional methods for producing educational material in histopathology. The described slide scanning and viewing technology will also enable novel applications, such as large scale quality assurance programmes, virtual slide seminars, and virtual slides as supplements to scientific publications. In this editorial, we describe current and future applications of virtual microscopy, and we discuss how the technology could be refined to allow even further applications in diagnostic pathology.

\section{"Virtual slides archived on a net- work server can be individually controlled and viewed by a large number of simultaneous users"}

The terms "virtual microscopy" and "telepathology" are often confused. Dynamic telepathology refers to remote robotic operation of a motorised microscope and real time transmission of the video image. ${ }^{2}$ In contrast, virtual microscopy is a form of static telepathology, where digital imaging technology is used to digitise, store, and view slides. A digital representation of an entire slide at the resolution of a high magnification objective is designated a "virtual slide". The application spectrum of dynamic telepathology is limited by its "live" nature and single user control, whereas virtual slides archived on a network server can be individually controlled and viewed by a large number of simultaneous users. This is the key to a wide range of potential applications of virtual microscopy, from production of educational material to integration into the hospital patient record system.

\section{APPLICATIONS}

\section{Education}

Educational material in pathology teaching has traditionally been based on printed micrographs and projection slides, and buyers of modern textbooks now often receive a supplemental CD with digital images. Whether digital or not, an ordinary micrograph is limited to a preselected area and magnification. In contrast, virtual microscopy enables viewing of any part of a specimen at any magnification. With annotations, selected important areas in a virtual slide can be pinpointed, corresponding to micrographs in printed textbooks. In addition, for pathologists in training, virtual slide atlases provide a feasible way to view collections of very rare diagnostic entities. "Quiz modes" and other interactive functions, such as grading tutorials, are new and interesting educational tools.

\section{Research}

By providing virtual slides as supplements to research articles in scientific journals, results can be documented and thus verified by other researchers in a way not currently possible with ordinary printed micrographs. In molecular pathology research, studies using tissue microarrays could provide the entire study material viewable as supplemental web based virtual slides. Readers of case studies and studies describing new or rare histopathological entities will benefit from the possibility of viewing the virtual slides of the lesions described. Thus, virtual microscopy responds to increasing demands on openness and transparency in scientific reporting. Standard platforms for publishing virtual slides online, ensuring future access, would be valuable for authors, journals, and the readers.

\section{Live presentations}

Didactic live presentations on diagnostic pathology are currently largely dependent on the use of conventional digital images embedded in PowerPoint presentations. With virtual microscopy, the presenter, with the use of a mouse, can navigate to any area within a slide on the projector screen and seamlessly change magnification. Interesting areas that have been annotated beforehand can instantly be displayed. The visual experience of the audience thus resembles that of viewing a glass slide using a multiheaded microscope or a microscope connected to a projector, but the number of participants can be much larger. When it comes to clinical meetings or slide seminars, it is also possible for the audience to view the virtual slides before the presentation. It is easy to foresee that this will be an integral part of future pathology congresses, where the paid registration fee not only includes the congress abstracts, but also opens access to the congress website, containing virtual slides of the cases to be presented in the lectures.

\section{Multiple copies of microscope slides}

Applications requiring multiple copies of microscope slides are ideally suited to virtual microscopy. Interlaboratory comparisons of histopathological diagnoses have been conducted by preparing replicate sets of microscope glass slides and distributing them to the participating pathologists. When the specimen sample is small, such as a needle biopsy, this is not possible, and for cytological specimens there is often only a single diagnostic slide. Virtual microscopy allows each participant to view exactly the same tissue section, thus facilitating quality control programmes, even at a nationwide or international scale. Virtual microscopy could also be used for pathology board certification examinations, which are difficult to arrange if identical specimen sets are considered necessary.

\section{ISSUES INVOLVED}

A common feature of the applications discussed thus far is that virtual microscopy introduces a new level of functionality, not previously possible to accomplish. However, for further applications in diagnostic pathology, important issues to consider include image quality, slide navigation and viewing speed, depth of focus, storage costs, and scanning time.

The atlas presented in this issue of JCP shows that slides can be digitised with an image quality that would satisfy most pathologists. However, the image quality seen by the end user is ultimately determined by the resolution and size of the computer screen. The quality of monitors has improved rapidly, and one of the main improvements in the next generation of computer operating systems will be a shift from low resolution systems towards high resolution screen standards. With a large monitor, more image information can then be conveyed through a screen than is seen in one high power field through the oculars. ${ }^{3}$ Because each monitor and graphics adapter displays the image slightly differently, the possibility of directly adjusting visual properties such as brightness and contrast of the virtual slides is important, especially 
because every pathologist has his own personal way of fine tuning the settings in the microscope.

"The depth of field in a virtual slide can be extended by capturing and layering multiple focal planes, and these can also be digitally merged"

Navigation by stage movement becomes almost a reflex action in pathologists, but getting accustomed to navigating a virtual slide with the mouse in combination with a overview window offers great advantages. Within a standard intranet of a pathology department, the viewing system described in this issue corresponds well to conventional microscopy in terms of viewing speed. There is no noticeable time delay when zooming in and out (switching objectives), and the specimen is automatically in focus. However, in a microscope, it is possible to adjust the focus continually by moving the zone of sharpness up and down the optical axis. This is important, because with a high quality objective, the depth of field is smaller than the thickness of most histological specimens. ${ }^{3}$ The depth of field in a virtual slide can be extended by capturing and layering multiple focal planes, and these can also be digitally merged. This technology will be important especially for viewing cytological specimens, which represent entire cells, unlike $2-5 \mu \mathrm{m}$ thick tissue sections.

The resolution and image quality of the atlas slides described in this issue of $J C P$ were adjusted according to feedback from pathologists. This generated file sizes that only a few years ago would have resulted in massive storage costs. As a result of increasing capacity and falling prices of fast storage systems this is no longer a crucial issue, even if a department scanned every diagnostic slide produced in the laboratory. However, scanning with a $\times 40$ microscope objective was found necessary, leading to relatively long scanning times. ${ }^{1}$ Slide scanning with a $\times 10$ or $\times 20$ objective would be much faster, but the resulting image quality is clearly compromised. A promising technique to speed up scanning is parallel imaging using array microscope technology. ${ }^{4}$

Most of the discussed problems have thus already been solved, and it seems clear that all technical obstacles will eventually be solved. As discussed, storage costs are already manageable, and obtaining the scanning equipment is a one time cost. But although economically feasible, high quality digitisation of slides with current scanning systems is time consuming, so that its application in everyday diagnostic pathology cannot yet be recommended. Furthermore, the need for routine scanning of all diagnostic slides is still unclear. However, there is no doubt that a scanned copy of a slide would be valuable, because previous and current biopsies from the same patient could quickly be viewed, and slides would be readily available for meetings and consultations. It is likely that scanning of slides in pathology departments will begin with only the "interesting" slides being scanned as part of clinical meetings, consultations, and slide seminars. Regardless of whether all or only a part of all slides are scanned, it will be important that virtual slides are properly archived, together with complementary data, such as meeting protocols or seminar handouts, because these will constitute a valuable source of educational and reference material. There will gradually be a need to integrate the virtual slides into the hospital patient record system, thus creating a system corresponding to the highly successful picture archiving and communication systems in radiology departments.

\section{THE FINAL OBSTACLE}

The perception of an image also involves a non-conscious part in the sequence leading to diagnosis. Whether a pathologist used to conventional glass slides can get the same perception from a computer screen remains to be seen when all technical aspects are solved. Perhaps future pathologists, who will get a considerable part of their training from virtual slides, will develop a different kind of "feeling" for screen viewing. Perhaps there might be differences within the slides, some being better visualised on a computer screen, some using a microscope.

"The feedback that we have
received from pathologists shows
that virtual microscopy should cur-
rently be regarded as a useful
complement to conventional micro-
scopy"

Already today virtual microscopy has a high potential for various tasks in pathology education, in clinical meeting activity, and in quality control. The feedback that we have received from pathologists shows that virtual microscopy should currently be regarded as a useful complement to conventional microscopy. The digital revolution in pathology will not be as swift and radical as in radiology. Instead, it will take place in a stepwise fashion, the pace and applications involved dictated by the needs of the pathologists, and paralleled by advances in microscope slide scanning and computer monitor technology.

J Clin Pathol 2004:57:1250-1251. doi: 10.1136/jicp.2004.019919

\section{Authors' affiliations}

$J$ Isola, Institute of Medical Technology, University and University Hospital of Tampere, 33014 Tampere, Finland

M Lundin, J Lundin, Biomedical Informatics Group, Department of Oncology, University of Helsinki, FIN-00290 Helsinki, Finland

Correspondence to: $\operatorname{Dr} M$ Lundin, Biomedical Informatics Group, University of Helsinki, HUCH Clinical Research Institute, PO Box 105, FIN-00290 Helsinki, Finland; mikael.lundin@ helsinki.fi

\section{REFERENCES}

1 Lundin $\mathrm{M}$, Lundin J, Helin $\mathrm{H}$, et al. A digital atlas of breast histopathology: an application of webbased virtual microscopy. J Clin Pathol 2004; 57:1288-91

2 Cross SS, Dennis T, Start RD. Telepathology: current status and future prospects in diagnostic histopathology. Histopathology 2002;41:91-109.

3 Supplemental information (http:// www.WebMicroscope.net/breastatlas/ documentation/).

4 DMetrix, Inc. http://www.dmetrix.net/ (last accessed on 06/2004) 